\title{
Sintomatologia Depressiva, Estresse e Ansiedade em Universitários
}

\author{
Marília Guimarães Leal Jardim ${ }^{1}$ \\ Tathyane Silva Castro ${ }^{1}$ \\ Carla Fernanda Ferreira-Rodrigues ${ }^{1}$ \\ ${ }^{1}$ Universidade Federal do Vale do São Francisco, Petrolina, Pernambuco, Brasil
}

\begin{abstract}
Resumo
Os níveis de sintomatologia depressiva, estresse e ansiedade têm crescido em todo o mundo comprometendo a qualidade de vida e bem estar das pessoas das mais diferentes idades. Assim, o presente estudo teve como objetivo avaliar os níveis desses sintomas em 410 estudantes (233 ingressantes e 177 concluintes) dos cursos de saúde de uma universidade pública federal do interior de Pernambuco, a fim de verificar se estar no início ou final do curso pode interferir em tais níveis. Foram utilizados os seguintes instrumentos: questionário sociodemográfico, IDATE, EBADEP, EPS-10 e SRQ-20 em aplicações coletivas em sala de aula. Os resultados apresentaram indícios de sofrimento mental (53,9\%), prevalência de ansiedade (43,4\% ansiedade-estado; $42 \%$ ansiedade-traço), níveis de estresse moderado ( $M=20,68$ ingressantes; $M=20,31$ concluintes) e baixos índices de sintomatologia depressiva $(0,6 \%)$. Não foi identificada diferença estatisticamente significativa quanto aos construtos em relação ao início e ao final de curso, demonstrando que o ambiente universitário parece não ser adoecedor, mas outras variáveis devem ser investigadas, pois podem estar relacionadas a essas características nesse período da vida.
\end{abstract}

Palavras-chave: saúde mental, inventários, avaliação psicológica, jovens

Depressive Symptomatology, Stress and Anxiety in University Students

\begin{abstract}
The levels of depressive symptoms, stress, and anxiety have grown all over the world compromising the quality of life and wellbeing of people of a wide age range. Thus, this study aimed to evaluate the levels of these symptoms in 410 students (233 were beginners and 177 were graduating students) of a federal public university in the countryside of the state of Pernambuco, to verify whether being at the beginning or end of the course may interfere in such levels. The following instruments were used: sociodemographic questionnaire, IDATE, EBADEP, EPS-10, and SRQ-20 collectively applied in the classroom. The results showed signs of mental suffering (53.9\%), prevalence of anxiety ( $43.4 \%$ anxiety-state, $42 \%$ anxiety-trace), moderate stress levels ( $M=20.68$ beginners, $M=20.31$ graduating), and low rates of depressive symptoms $(0.6 \%)$. No statistically significant difference was identified with regard to the constructs considering the beginning and end of the course, showing that the university environment seems not to be unhealthy, but other variables should be investigated that may be related to that period. Keywords: mental health; inventories; psychological assessment; young adults.
\end{abstract}

Sintomatología Depresiva, Estrés y Ansiedad en Universitarios

\begin{abstract}
Resumen
Los niveles de sintomatología depresiva, estrés y ansiedad han aumentado en todo el mundo, comprometiendo la calidad de vida y el bienestar de personas de edades distintas. Así, el presente estudio tuvo como objetivo evaluar los niveles de estos síntomas en 410 alumnos de los cursos de salud de una universidad pública federal en el interior de Pernambuco, con el fin de verificar si el comienzo o el final del curso interfieren en dichos niveles. De estos 233 eran principiantes y 177 concluyentes. Se utilizaron los siguientes instrumentos: cuestionario sociodemográfico, IDATE, EBADEP, EPS-10 y SRQ-20 en aplicaciones colectivas en clase. Los resultados mostraron indicios de sufrimiento mental (53,9\%), prevalencia de ansiedad (43,4\% ansiedad-estado, 42\% ansiedad-traza), niveles de estrés moderado ( $M=20,68$ principiantes, $M=20,31$ concluyentes) y bajos índices de sintomatología depresiva $(0,6 \%)$. No se identificó diferencia estadísticamente significativa en cuanto a los constructos con relación al inicio y final de curso, demostrando que el ambiente universitario parece no causar enfermedades, pero se deben investigar otras variables, ya que pueden estar relacionadas con estas características en este período de la vida.

Palabras clave: salud mental; inventarios; evaluación psicológica; jóvenes.
\end{abstract}

A tríade depressão, estresse e ansiedade vêm crescendo de maneira exponencial e interessando os pesquisadores. O estresse é apontado como epidemia global. A depressão atinge $5,8 \%$ da população brasileira, sendo uma das maiores causas de incapacitação, e os distúrbios relacionados a ansiedade afetam 9,3\% das pessoas que vivem no Brasil (OMS, 2017).
Depressão, estresse e ansiedade podem atingir qualquer pessoa. No entanto, a sintomatologia associada a esses tem maior chance de surgir pela primeira vez no início da vida adulta, principalmente no período universitário (Figueiredo et al., 2014). As pesquisas estudam esse sofrimento mental e falam acerca desse contexto de propensão ao sofrimento psíquico 
dos universitários. Os estudantes tendem a manifestar algum transtorno durante sua vida acadêmica, sendo o de ansiedade o mais recorrente (Carvalho, Bertolini, Milani, \& Martins, 2015; Cavestro \& Rocha, 2006; Cerchiari, Caetano, \& Faccenda, 2005; Fonseca, Coutinho, \& Azevedo, 2008; Oliveira, 2013).

A literatura versa sobre os motivos pelos quais existe alta prevalência nos estudantes. Primeiramente, pode-se citar que o ingresso na universidade é um momento de transformações, no qual o jovem precisa se adaptar a uma nova forma de ensino e aprendizagem, onde também ocorre a formação de novas vinculações, entre outras mudanças. $\mathrm{O}$ aumento dessas novas demandas pode gerar insegurança e conflitos que podem causar ansiedade, estresse e depressão (Almeida \& Soares, 2003; Lacerda, 2015; Martincowski, 2013). Tanto a entrada na universidade pode ser disparadora desses sintomas, quanto à saída desse ambiente também é um agravador. Nesse caso, sair do ambiente acadêmico para o mercado do trabalho envolve lidar com incertezas e dificuldades que requerem mais qualidades e atributos do indivíduo. Ou seja, ao se deparar com o mercado de trabalho, o jovem, agora profissional, precisa estar ainda mais capacitado e apto para enfrentar a concorrência no mercado (Assis, Silva, Lopes, Silva, \& Santini, 2013).

Para Marchi, Bárbaro, Miasso e Tirapelli (2013), os estudantes da área de saúde são os que apresentam mais sintomas ansiosos, depressivos e de estresse. Dentre esses, a depressão é apontada como o transtorno de maior prevalência (Vasconcelos et al., 2015; Paula et al., 2014). Os cursos da área de saúde possuem alguns fatores desencadeantes de sintomas, como experiência da prática clínica, o lidar com o ser humano, o contato com o sofrimento psíquico, a observação constante dos instrutores no cenário da prática, o medo de cometer erros e sentimentos de inadequação. Além disso, ainda contam com uma grade curricular integral, o que exige ainda mais dedicação e acarreta menos tempo para atividades fora da universidade (Lima, Domingues, \& Cerqueira, 2006; Rocha \& Sassi, 2013).

Há pesquisas com universitários que verificaram diferenças significativas em relação à prevalência de tais construtos nos ingressantes (Alvi et al., 2010; Brandtner \& Bardagi, 2009; Carvalho et al., 2015) e outras verificaram diferença preponderante da metade para o final do curso (Draken, Hafen, Rush, \& Reisbig, 2012; Simić-Vukomanovic et al., 2016). Apesar disso, há sempre um ponto em comum, que é a concordância acerca do sofrimento mental e da vulnerabilidade do estudante universitário. Ademais, tanto para os ingressantes quanto para os concluintes, são exigidas adaptações para experienciar novas situações (Carvalho et al., 2015; Cavestro \& Rocha, 2006; Rodrigues, Marques, Neto, Montesinos, \& Oliveira, 2016). Essa vulnerabilidade fica ainda mais clara quando se verifica a alta correlação que existe entre depressão, estresse e ansiedade em universitários (Apóstolo, Figueiredo, Mendes, \& Rodrigues, 2011; Vasconcelos et al., 2015).

Um fator de destaque em relação a prevalência de sintomatologia depressiva, estresse e ansiedade é a ligação com o sexo dos indivíduos. Os estudos endossam a discussão e apontam para uma prevalência feminina, de forma que tais sintomatologias acometem mais as mulheres, não só dentre as que estão na universidade, como também em relação àquelas que desempenham outras atividades na sociedade em geral. Algumas atribuições, geralmente, estão mais associadas ao contexto de vida feminino, como sobrecarga de trabalho (trabalhar por longos períodos, sem ajuda de outrem), além de jornadas duplas. A presença destas entre outras atribuições no ambiente feminino e não no masculino podem vir a se tornar desencadeadoras desses sintomas, favorecendo assim a prevalência nas mulheres (Araújo, Pinho, \& Almeida, 2005; Fioravanti, Santos, Maissonette, Cruz, \& Fernandez, 2006; Monteiro, Tavares, \& Pereira, 2008; Pinho \& Araújo, 2012; Santos, Almeida, Martins, \& Moreno, 2003).

Outro ponto envolve as variáveis pessoais que podem prejudicar a saúde mental desses universitários (Almeida \& Soares, 2003). Essas variáveis dizem respeito ao afastamento familiar, gestão do dinheiro (que nesse momento a família envia e o jovem administra) e formação de novos vínculos sociais. Assim, chama-se atenção para o fato de que morar com a família é um fator de proteção à saúde mental, pois no seio familiar esse jovem dispõe de pessoas em que confia e de amigos com os quais já possui vínculo formado. Além disso, junto com sua família, esse jovem, muitas vezes, ainda está em um lugar mais passivo, ao contrário do momento em que precisa sair de sua casa, tendo então que tomar uma postura mais ativa, adaptar seus comportamentos e se encaixar nesse novo espaço (Alves, 2014; Sousa, José, \& Barbosa, 2013).

Assim como morar na casa da família pode ser um fator de proteção, a literatura indica que a prática de exercícios de forma regular também pode influenciar a saúde mental (Vieira \& Schermann, 2015). Outro fator protetivo é o contentamento com a opção acadêmica. 
Essa variável influencia diretamente o desempenho desses estudantes. Assim, o estudante que, por algum motivo, não esteja satisfeito com seu curso pode sofrer impacto na sua saúde mental e nas relações que estabelece (Bardagi, 2007; Ramos et al., 2015).

Os dados apresentados acima se tornam ainda mais preocupantes pelo fato de, muitas vezes, o estudante em sofrimento não procurar por apoio psicológico especializado (Cerchiariet al., 2005). Assim, é extremamente importante discutir e fomentar pesquisas nessa área, para que a gestão acadêmica possa criar mecanismos de cuidados para esses estudantes.

Diante do exposto, esta pesquisa teve por objetivo verificar os níveis de ansiedade, estresse e sintomatologia depressiva de estudantes dos cursos da área de saúde de uma universidade pública federal em início e fim de curso com o intuito de verificar se o fato de estar em início ou fim de curso pode influenciar tais níveis. Ademais, buscou-se verificar a relação entre os construtos, possíveis diferenças entre sexo e as peculiaridades socioeconômicas da amostra investigada.

\section{Método}

\section{Participantes}

A pesquisa contou com uma população amostral de 410 universitários divididos entre ingressantes $(N=233 ; 56,8 \%)$ e concluintes $(N=177 ; 43,2 \%)$ de quatro cursos da área de saúde de uma universidade pública federal localizada na cidade de Petrolina-PE. Do total, a maioria era do sexo feminino $(N=259$; $63,3 \%)$, com idade média de 23,59 anos $(D P=5,61)$ variando de 18 a 63 anos. Em relação ao curso universitário, a maior parte da amostra foi do curso de Psicologia ( $N=134 ; 32,68 \%)$, seguidos por Medicina $(N=107 ; 26,10 \%)$, Enfermagem $(N=87 ; 21,22 \%)$ e Farmácia $(N=82 ; 20 \%)$.

Foram considerados ingressantes os estudantes do $1^{\circ}$ período em todos os cursos, e como concluintes os estudantes do $9^{\circ}$ e $10^{\circ}$ períodos de Psicologia, e do $8^{\circ}$ período nos cursos de Medicina, Enfermagem e Farmácia. Essa diferenciação nos períodos de conclusão aconteceu pelo fato de que as turmas de Psicologia, ainda no $9^{\circ}$ e $10^{\circ}$ períodos tinham aulas em sala. $O$ mesmo não acontece nos demais cursos pesquisados, pois nesses períodos finais os estudantes estão em atividades práticas fora da universidade, o que dificulta o acesso a eles. Sendo assim, optou-se por considerar como concluintes os estudantes do $8^{\circ}$ período, ou seja, aqueles que cursavam o último semestre de aulas teóricas, antes de irem para o estágio final (prática) do curso.

Foram excluídos da amostra os estudantes menores de 18 anos. A coleta de dados ocorreu com os estudantes matriculados no segundo semestre de 2017 e no primeiro semestre de 2018.

\section{Instrumentos}

Os estudantes responderam os instrumentos a seguir:

Questionário sociodemográfico. Questionário elaborado para caracterização da amostra, utilizado para rastreio do perfil social, demográfico e econômico dos participantes. Estavam incluídos dados como sexo, idade, moradia, renda familiar, atividade de trabalho e outros.

Inventário de Ansiedade Traço-Estado (IDATE). Questionário autoaplicável, composto por duas subescalas: IDATE-E, para avaliar a ansiedade enquanto estado, e IDATE-T, utilizada para avaliar a ansiedade como traço. Cada escala é constituída de 20 itens, respondidos em uma escala do tipo Likert, variando de um a quatro. Essa variação vai de "absolutamente não" a "muitíssimo" para a subescala IDATE-E, e de "quase nunca" a "quase sempre" para subescala IDATE-T. O escore total individual de cada escala varia de 20 a 80 pontos (Biaggio \& Natalício, 2003; Fioravanti et al., 2006).

Escala de Estresse Percebido (EPS-10). Essa escala foi utilizada para medir o estresse percebido pelo sujeito no último mês. As questões versam a respeito dos sentimentos e pensamentos dos sujeitos durante esse período. Possui 10 itens do tipo Likert, com escores variando de zero (nunca) a quatro (sempre). O escore total do instrumento pode variar de zero a 40 pontos (Reis, Hino, \& Añez, 2010).

Escala Baptista de Depressão (Versão Adulto) - EBADEP-A. Instrumento autoaplicável que tem por objetivo o rastreamento de sintomatologia depressiva. É composta por 90 frases, apresentada em pares, formando 45 itens. Cada item apresenta um indicador de sintomatologia com uma frase de cunho positivo e outra de cunho negativo. A EBADEP-A tem respostas do tipo Likert de quatro pontos, variando de zero a três, com pontuação mínima de zero e máxima de 135 pontos. Para interpretá-la, considera-se que quanto menor a pontuação, menor é a sintomatologia em depressão (Baptista \& Gomes, 2011).

Self Report Questionnaire (SRQ-20). Instrumento que avalia o sofrimento mental (transtornos 
mentais não psicóticos). As respostas são do tipo sim/ não. Cada resposta "sim" vale um ponto e a soma total corresponde ao escore final. A pontuação obtida se relaciona com a probabilidade de presença de transtorno não psicótico, variando de zero (nenhuma probabilidade) a 20 (extrema probabilidade). Para estabelecer faixas que indiquem a existência ou não de sofrimento mental, foi adotado que quando o resultado for $\geq 7$ (maior ou igual a sete respostas SIM) indica probabilidade de sofrimento mental (Gonçalves, Stein, \& Kapczinski, 2008; Padovani et al., 2014).

\section{Procedimentos}

A pesquisa foi submetida e aprovada pelo Comitê de Ética em Pesquisa (CEP) da Universidade Federal do Vale do São Francisco - Univasf sob o número do parecer: CAEE No 80541217.6.0000.5196, respeitando os princípios éticos da resolução 510/2016 do Ministério da Saúde. Após a autorização do professor, os estudantes foram contatados durante as aulas para responder os instrumentos da pesquisa de forma coletiva em sala de aula. Os questionários foram respondidos em um tempo aproximado de 40 minutos.

\section{Análise dos Dados}

Os dados foram analisados utilizando o software Statistical Package for Social Sciences (SPSS), versão 20. Foram realizadas estatísticas descritivas para melhor caracterizar a amostra. Para comparar os construtos investigados nos estudantes ingressantes e concluintes, bem como em relação ao sexo, foi realizado o teste $t$ de Student. Também foi realizada uma correlação de Pearson a fim de verificar a relação entre a sintomatologia depressiva, estresse e ansiedade. Para verificar a prevalência dos construtos investigados na amostra, foram considerados os escores e os pontos de corte de cada instrumento utilizado.

\section{Resultados}

Inicialmente serão descritos os dados sociodemográficos. Em seguida serão apresentados os resultados obtidos sobre a sintomatologia depressiva, estresse e ansiedade nos estudantes, de maneira geral, e comparando os resultados entre sexo, ingressantes/ concluintes e por curso.

Dos 410 universitários que participaram do estudo, houve um equilíbrio entre os que precisaram mudar de cidade para cursar a universidade $(50,2 \%)$ e os que não precisaram realizar essa mudança $(49,8 \%)$.
Apenas no curso de Medicina obteve-se uma proporção maior $(68,2 \%)$ entre os que precisaram mudar de cidade. Os participantes relataram morar com a família, que incluía pais e irmãos (23,5\%), 22,5\% moravam somente com os pais, totalizando $46 \%$ dos entrevistados que dividiam a moradia com familiares (pais, mães e irmãos); seguidos por $17,6 \%$ que moravam com amigos; $15,2 \%$ moravam sozinhos; $7,8 \%$, com irmãos; $7,6 \%$, com outros parentes; $4,7 \%$, com maridos/esposas e $1 \%$ com filhos.

Com relação ao perfil econômico dos participantes, a grande maioria $(N=386 ; 94,9 \%)$ é dependente economicamente. Em relação ao desenvolvimento de atividades remuneradas apenas 15,1\% $(N=62)$ desenvolviam alguma atividade. Quanto ao recebimento de benefícios sociais, a minoria $(N=43 ; 10,6 \%)$ afirmou receber algum auxílio. Sobre a renda familiar, em salários mínimos, os participantes estavam distribuídos da seguinte forma: 20 informaram não ter nenhuma renda (4,9\%), 138 estavam na faixa de um a dois salários $(33,9 \%), 116$ entre três a quatro salários $(28,5 \%), 66$ entre cinco a seis salários $(16,2 \%), 30$ entre sete a oito salários $(7,4 \%), 17$ entre nove a 10 salários $(4,2 \%)$ e 20 informaram renda acima de 10 salários (4,9\%).

Sobre os aspectos relacionados à saúde, a pesquisa mostrou que $58,6 \%$ do total dos estudantes não possuíam plano de assistência médica. No entanto, os acadêmicos de Medicina apareceram divergindo do percentual total, visto que $53,3 \%$ afirmaram possuir plano de saúde. Ainda sobre à saúde, 55,8\% dos participantes não praticavam nenhuma atividade física. Fazendo uma comparação entre os estudantes de cada curso, os que mais afirmaram realizar atividades físicas foram os de Medicina (56,1\%), seguido por Psicologia (46,3\%), Enfermagem (38,4\%) e Farmácia (31,2\%). Dentre os participantes, 181 (44,3\%) afirmaram ter buscado ajuda psicológica. Entre aqueles que já buscaram ajuda de um profissional de Psicologia, 26,5\% trazem como queixa principal a ansiedade e $7,2 \%$ trazem a ansiedade em associação com outros fatores. Sintomas depressivos vêm em segundo lugar, com um percentual de $12 \%$ daqueles que buscaram auxílio psicológico. Um total de 55 participantes $(13,5 \%)$ também afirmaram já ter procurado ajuda psiquiátrica.

A respeito dos dados acadêmicos dos participantes, foi encontrado que $70,9 \%$ estão em sua primeira graduação. Não foram especificados quantos, dos que não estavam cursando o ensino superior pela primeira vez, concluíram seu primeiro curso superior. A maioria dos participantes $(69,2 \%)$ afirmou que a escolha 
do curso está ligada a realização pessoal, sendo um resultado comum a todos os cursos pesquisados. Outros motivos para a escolha do curso referem-se à influência de familiares $(8,8 \%)$, remuneração financeira $(1,2 \%)$, dois desses fatores associados $(5,42 \%)$ e outros motivos (15,2\%). Em relação à satisfação com o curso, $90 \%$ dos ingressantes afirmaram estar satisfeitos e, entre os concluintes, esse percentual é de $86,9 \%$. Foi solicitado que os estudantes indicassem uma nota de zero a dez, correspondente ao nível de satisfação com o curso escolhido. Como pode ser observada na Tabela 1 , a maior média de satisfação foi no curso de Medicina com 8,43; seguido pelos estudantes de Psicologia com 8,33; Enfermagem com 7,84 e Farmácia com 7,81. Pode-se observar também que entre os participantes ingressantes, $33,2 \%$ já pensaram em desistir do curso, enquanto que entre os concluintes esse índice sobe para 46,9\%.

Outro dado importante é que dentro da amostra, 320 participantes $(78,4 \%)$ afirmaram deixar de fazer coisas prazerosas por causa da universidade. Entre os participantes por curso os percentuais são de: $75,2 \%$ no curso de Psicologia; $72 \%$ no curso de Medicina; $85,1 \%$ no curso de Enfermagem e 85,2\% no curso de Farmácia.

$\mathrm{Na}$ Tabela 2 estão descritos os escores médios dos estudantes por curso para ansiedade, estresse, sintomatologia depressiva e sofrimento mental, bem como os valores máximos e mínimos encontrados para cada grupo. Ao comparar as médias dos cursos em cada um dos construtos, verificou-se que o curso de Medicina difere significativamente $(p \leq 0,05)$ de todos os outros cursos, apresentando índices menores de ansiedade, estresse, sintomatologia depressiva e sofrimento mental.

O primeiro construto analisado foi a ansiedade por meio de duas escalas que mediram o estado e o traço de ansiedade nos participantes. Quando esse construto foi relacionado em faixas de sintomatologia leve, moderada e alta, a maioria dos participantes apresentou uma sintomatologia moderada. Para a ansiedade estado 9,2\% (N=36) apresentaram ausência ou sintomatologia leve; $47,4 \%(N=186)$ sintomatologia moderada e $43,4 \%(N=170)$ sintomatologia alta. Para a ansiedade traço $12,3 \%(N=49)$ apresentaram ausência ou sintomatologia leve; $45,7 \%(N=182)$ sintomatologia moderada e $42 \%(N=167)$ altos índices de sintomatologia. Para estabelecer os pontos de corte (leve, moderada e alta) utilizados nesse artigo foram considerados os adotados anteriormente na literatura (Agostini, Sakae, \& Feldens, 2010; Santos, Cendoroglo, \& Santos, 2017). A utilização de pontos de corte já encontrados na literatura justifica-se pelo uso em contexto de pesquisa, não sendo utilizado para fins de diagnóstico clínico. O teste qui-quadrado de aderência indicou tanto para ansiedade traço quanto estado que os dados não são consistentes nas três faixas de sintomatologia, ou seja, a amostra distribuiu-se de maneira homogênea apenas entre a faixa moderada e alta de ansiedade $\left(X^{2}(2)=103,85 ; p \leq 0,05\right)$.

$\mathrm{O}$ segundo construto analisado foi o estresse. Em todos os cursos as médias de estresse apresentaram

Tabela 1.

Nivel de Satisfação dos Estudantes com o Curso

\begin{tabular}{lccccc}
\hline & & Psicologia & Medicina & Enfermagem & Farmácia \\
\hline $\begin{array}{l}\text { Satisfeito com } \\
\text { o curso }\end{array}$ & Sim & 126 & 98 & 68 & 68 \\
& & $(94,7 \%)$ & $(91,6 \%)$ & $(80 \%)$ & $(84 \%)$ \\
& Não & 7 & 9 & 17 & 13 \\
& & $(5,3 \%)$ & $(8,4 \%)$ & $(20 \%)$ & $(16 \%)$ \\
& Total & 133 & 107 & 85 & 81 \\
Já pensou em & \multirow{2}{*}{ Sim } & $(100 \%)$ & $(100 \%)$ & $(100 \%)$ & $(100 \%)$ \\
desistir & & 49 & 26 & 51 & 33 \\
& Não & $(36,8 \%)$ & $(24,3 \%)$ & $(59,3 \%)$ & $(40,7 \%)$ \\
& & 84 & $(75,7 \%)$ & $(40,7 \%)$ & 48 \\
& Total & $(63,2 \%)$ & 107 & 86 & $(59,3 \%)$ \\
& & $(100 \%)$ & $(100 \%)$ & $(100 \%)$ & 81 \\
& & & & $(100 \%)$ \\
\hline
\end{tabular}


Tabela 2.

Escores dos Construtos por Curso

\begin{tabular}{lcccccc}
\hline & Curso & N & Mínimo & Máximo & Média & $D P$ \\
\hline Ansiedade Estado & Psicologia & 126 & 26 & 73 & 47,29 & 10,47 \\
& Medicina & 103 & 22 & 70 & 43,70 & 10,81 \\
& Enfermagem & 84 & 22 & 77 & 50,01 & 11,45 \\
& Farmácia & 79 & 27 & 74 & 50,76 & 9,80 \\
Ansiedade Traço & Psicologia & 129 & 21 & 75 & 47,56 & 11,86 \\
& Medicina & 104 & 24 & 68 & 42,75 & 10,59 \\
& Enfermagem & 85 & 23 & 72 & 49,28 & 11,58 \\
Estresse & Farmácia & 80 & 28 & 74 & 48,54 & 10,18 \\
& Psicologia & 130 & 5 & 36 & 20,64 & 7,05 \\
& Medicina & 107 & 2 & 34 & 17,47 & 7,07 \\
Sintomatologia & Enfermagem & 84 & 4 & 37 & 22,85 & 6,85 \\
Depressiva & Farmácia & 79 & 5 & 36 & 21,96 & 6,24 \\
& Psicologia & 105 & 2 & 120 & 45,88 & 24,11 \\
& Medicina & 95 & 0 & 93 & 36,56 & 22,21 \\
Sofrimento Mental & Enfermagem & 68 & 6 & 126 & 45,94 & 24,71 \\
& Farmácia & 66 & 4 & 92 & 46,09 & 22,46 \\
& Psicologia & 133 & 0 & 17 & 7,67 & 4,64 \\
& Medicina & 106 & 0 & 20 & 5,91 & 4,74 \\
& Enfermagem & 84 & 0 & 20 & 8,73 & 4,87 \\
& Farmácia & 78 & 0 & 19 & 7,86 & 4,51 \\
\hline
\end{tabular}

valores moderados: $M=20,64(D P=7,05)$ Psicologia; $M=17,47(D P=7,07)$ Medicina; $M=22,85(D P$ $=6,85)$ Enfermagem e $M=21,96(D P=6,24)$ Farmácia. Em relação à sintomatologia depressiva nos cursos, aquele que apresentou maior média foi Farmácia. Entre os participantes $89,2 \%(N=298)$ estão na faixa que indica ausência ou sintomatologia depressiva leve; $10,2 \%(N=34)$ estão na faixa de sintomatologia moderada e $0,6 \%(N=2)$ na sintomatologia severa. $\mathrm{O}$ teste qui-quadrado de aderência indicou que os dados não são consistentes com a distribuição especificada, ou seja, a maioria da amostra concentrou-se na sintomatologia depressiva leve $\left[X^{2}(2)=474,06 ; p \leq 0,05\right]$.

O último construto analisado foi o sofrimento mental dos participantes. Em relação aos resultados dos participantes, 46,1\% ( $N=185)$ obtiveram resultado negativo para sofrimento mental, enquanto que $53,9 \%$ $(N=216)$ obtiveram escores igual ou maior que sete, apresentando probabilidade de sofrimento psíquico. Vale salientar que apenas o uso desse instrumento não é suficiente para diagnósticos de existência de sofrimento mental.

$\mathrm{Na}$ Tabela 3, observam-se as médias de ansiedade, estresse, sintomatologia depressiva e sofrimento mental dos estudantes ingressantes e concluintes. Observa-se também por meio do Teste $t$ de Student realizado que as diferenças existentes entre os grupos não é estatisticamente significativa $(\phi \geq 0,05)$. Porém, ao comparar as médias em relação ao sexo, o desempenho entre os dois grupos revelou que houve diferença estatisticamente significativa $(p \leq 0,05)$ entre os sexos masculino e feminino em todos os construtos estudados. Assim, observou-se que as mulheres universitárias dessa amostra são mais ansiosas, além de exibirem mais sintomas de estresse, sintomatologia depressiva e sofrimento mental quando comparadas aos homens.

De acordo com os resultados apurados, pode-se observar que houve uma correlação positiva e significativa entre os construtos. Como pode ser visto na Tabela 
Tabela 3.

Comparação entre os Construtos por Etapa em que Está no Curso e por Sexo

\begin{tabular}{|c|c|c|c|c|c|c|}
\hline Construto & Grupo & $N$ & Média & $D P$ & $t$ & $p$ \\
\hline \multirow[t]{2}{*}{ Ansiedade Estado } & Ingressantes & 224 & 47,38 & 11,06 & $-0,50$ & 0,61 \\
\hline & Concluintes & 168 & 47,95 & 10,83 & & \\
\hline \multirow[t]{2}{*}{ Ansiedade Traço } & Ingressantes & 227 & 47,63 & 11,21 & 1,54 & 0,12 \\
\hline & Concluintes & 171 & 45,85 & 11,59 & & \\
\hline \multirow[t]{2}{*}{ Estresse } & Ingressantes & 223 & 20,68 & 7,14 & 0,51 & 0,61 \\
\hline & Concluintes & 177 & 20,31 & 7,12 & & \\
\hline \multirow{2}{*}{$\begin{array}{l}\text { Sintomatologia } \\
\text { Depressiva }\end{array}$} & Ingressantes & 194 & 43,13 & 23,16 & $-0,13$ & 0,89 \\
\hline & Concluintes & 140 & 43,49 & 24,43 & & \\
\hline \multirow[t]{2}{*}{ Sofrimento Mental } & Ingressantes & 224 & 7,37 & 4,80 & $-0,42$ & 0,67 \\
\hline & Concluintes & 177 & 7,58 & 4,80 & & \\
\hline \multirow[t]{2}{*}{ Ansiedade Estado } & Masculino & 142 & 44,73 & 11,50 & $-4,16$ & 0,00 \\
\hline & Feminino & 249 & 49,49 & 10,48 & & \\
\hline \multirow[t]{2}{*}{ Ansiedade Traço } & Masculino & 147 & 44,16 & 12,86 & $-3,75$ & 0,00 \\
\hline & Feminino & 250 & 48,69 & 10,77 & & \\
\hline \multirow[t]{2}{*}{ Estresse } & Masculino & 146 & 18,44 & 7,76 & $-4,65$ & 0,00 \\
\hline & Feminino & 253 & 21,86 & 6,62 & & \\
\hline \multirow{2}{*}{$\begin{array}{l}\text { Sintomatologia } \\
\text { Depressiva }\end{array}$} & Masculino & 129 & 38,82 & 21,85 & $-2,78$ & 0,00 \\
\hline & Feminino & 205 & 46,19 & 24,46 & & \\
\hline \multirow[t]{2}{*}{ Sofrimento Mental } & Masculino & 149 & 6,13 & 4,90 & $-4,39$ & 0,00 \\
\hline & Feminino & 251 & 8,29 & 4,65 & & \\
\hline
\end{tabular}

4 as correlações são fortes $(r \geq 0,70)$ o que indica que os construtos são altamente relacionados.

\section{Discussão}

O objetivo principal deste estudo foi verificar os níveis de sintomatologia depressiva, estresse, ansiedade e sofrimento mental em estudantes universitários da área de saúde em início e fim de curso. Os resultados não identificaram diferença significativa nos construtos investigados entre ingressantes e concluintes. Assim, parece que não são necessariamente questões acadêmicas que desencadeiam os sintomas aqui identificados, visto que a presença em atividades acadêmicas não os agravou. Nas últimas décadas, ocorreu uma mudança no acesso ao ensino superior no Brasil, sendo este ampliado e interiorizado (Mancebo, Vale, \& Martins, 2015; Marques, Cepêda, \& Zambello, 2015). As mudanças no acesso levaram a uma maior diversificação das características econômicas e sociais dos universitários (Neves, Raizer, \& Fachinetto, 2007).
Desse modo, pode-se sugerir que não parece ser o ambiente universitário de forma individual que é adoecedor, mas uma combinação de fatores, como a mudança de perfil dos estudantes que tem entrado no ensino superior, que contribuem com o aumento dessas sintomatologias. Sugere-se que outros fatores podem ser gatilhos para estresse e ansiedade e que os sintomas depressivos foram leves nessa amostra. Ademais, resultado semelhante foi encontrado na pesquisa de Cavestro e Rocha (2006) que demonstrou que não existiam diferenças relevantes entre os níveis de depressão e ansiedade nos estudantes de início e final do curso.

Entretanto, na literatura também existem estudos nos quais foram identificadas diferenças em relação aos ingressantes e concluintes (Carvalho et al., 2015) e que essa diferença já inicia da metade até o final do curso (Draken et al., 2012; Simić-Vukomanovic et al., 2016). Assis, Silva, Lopes, Silva e Santini (2013) afirmam que o fim do curso é o momento de maior estresse da vida acadêmica, em decorrência do estágio obrigatório, relatório de estágio, trabalho de conclusão de curso. Ainda 
Tabela 4.

Correlação entre os Construtos Ansiedade-Estado, Ansiedade Traço, Estresse, Sintomatologia Depressiva e Sofrimento Mental

\begin{tabular}{|c|c|c|c|c|c|c|}
\hline & & $\begin{array}{l}\text { Ansiedade } \\
\text { Estado }\end{array}$ & $\begin{array}{l}\text { Ansiedade } \\
\text { Traço }\end{array}$ & Estresse & $\begin{array}{c}\text { Sintomatologia } \\
\text { Depressiva }\end{array}$ & $\begin{array}{c}\text { Sofrimento } \\
\text { Mental }\end{array}$ \\
\hline \multirow[t]{2}{*}{ Ansiedade Estado } & $r$ & 1 & & & & \\
\hline & $N$ & 392 & & & & \\
\hline \multirow{2}{*}{ Ansiedade Traço } & $r$ & $0,73 * *$ & 1 & & & \\
\hline & $N$ & 381 & 398 & & & \\
\hline \multirow[t]{2}{*}{ Estresse } & $r$ & $0,74 * *$ & $0,81 * *$ & 1 & & \\
\hline & $N$ & 383 & 389 & 400 & & \\
\hline Sintomatologia & $r$ & $0,68 * *$ & $0,76^{* *}$ & $0,78^{* *}$ & 1 & \\
\hline Depressiva & $N$ & 318 & 326 & 326 & 334 & \\
\hline \multirow[t]{2}{*}{ Sofrimento Mental } & $r$ & $0,70 * *$ & $0,74 * *$ & $0,74^{* *}$ & $0,83 * *$ & 1 \\
\hline & $N$ & 385 & 391 & 392 & 327 & 401 \\
\hline
\end{tabular}

** Correlação significativa ano nível 0.01 .

assim, os autores concordam que tanto o ingresso quanto o egresso na vida universitária são agravadores de sintomas e somatizações, porém, no presente estudo, o ambiente acadêmico pareceu não ser o desencadeador desses sintomas tanto para o curso de Psicologia em que os estudantes estavam no último ano do curso, quanto para os de Farmácia, Medicina e Enfermagem que estavam no oitavo semestre do curso.

Analisando os construtos em relação ao sexo, foi verificado que há diferença estatisticamente significativa entre os grupos. As mulheres manifestaram maiores índices em comparação aos homens. Esse dado é corroborado com outros estudos que versam sobre o predomínio de sintomas de ansiedade e depressão em mulheres. Porém, não foram encontradas pesquisas sobre as sintomatologias especificamente no público de mulheres universitárias. Quando se fala das mulheres na sociedade, os estudos apontam o acúmulo de papéis atribuídos às mulheres. Por exemplo, as mulheres normalmente precisam conciliar suas atividades laborais com o trabalho doméstico, demanda que não acontece, de forma geral, com os homens. Outro ponto é o não compartilhamento e reconhecimento do exercício de atividades domésticas, a partir da falta de apoio dos outros membros da casa. Assim, a mulher tende a não ter tempo para atividades do lazer ou de cuidado para si mesma. Nota-se também a demanda de cuidado do outro que é requerida da mulher, e não acontece da mesma forma com indivíduos do sexo masculino (Pinho \& Araújo, 2012; Araújo et al., 2005).
No entanto, essa não parece ser a realidade da maioria das mulheres da amostra do estudo, visto que, no geral, são jovens, solteiras e dependente economicamente. Assim, pode-se atentar para outras variáveis que podem influenciar os índices mais elevados de ansiedade, sintomatologia depressiva e estresse nas mulheres. Keita (2007) destaca que as mulheres tendem a ser mais expostas a eventos negativos, como discriminação de gênero, abuso físico e sexual. Ainda, provavelmente influenciadas pela cultura, as mulheres se mostram mais sensíveis e abertas a falarem de sentimentos e percepções, além de serem mais críticas e observadoras. Em contrapartida, os homens ainda mostram receio em se expor, procurando menos serviços de ajuda (Fioravanti et al., 2006; Molina et al., 2014; Monteiro et al., 2008; Santos et al., 2003).

Um dado importante encontrado no estudo tem relação com a alta correlação entre os construtos ansiedade, depressão e estresse, indicando comorbidade, como é visto em outras pesquisas. Ou seja, ao passo que um construto aumenta, os outros tendem a aumentar, e isso evidencia a vulnerabilidade dos alunos à tríade de patologias (Vasconcelos et al., 2015). A forte associação entre esses construtos podem ser argumentadas pelo fato de que depressão, ansiedade e estresse parecem ser pontos distintos de um mesmo continuum. Sendo assim, essa tríade partilha alguns de seus sintomas trazendo uma forte relação intrínseca (Apóstolo et al., 2011).

Durante a formação acadêmica de $15 \%$ a $25 \%$ dos estudantes universitários apresentam algum transtorno, 
sendo o de ansiedade o mais comum (Carvalho et al., 2015; Cavestro \& Rocha, 2006). Desse modo, foram analisados os dados referentes a ansiedade geral (estado e traço) e nota-se que há uma alta prevalência de ansiedade nos estudantes da área de saúde.

Os estudantes de todos os cursos obtiveram médias com escores moderados para o estresse. Portanto, não foi identificado um índice alarmante de estresse nos sujeitos da pesquisa. O estresse, por sua vez, não é algo patológico, configurando-se como uma preparação do organismo frente a situações novas. Então se pode inferir que ambos os momentos analisados neste trabalho (ingresso e conclusão) requerem adaptação e novas habilidades (Rodrigues et al., 2016).

Quando analisadas as médias encontradas para estresse, identificou-se que a menor média foi no curso de Medicina. Também foram esses os estudantes que mais afirmaram praticar exercícios físicos de forma regular. Pode-se, então, hipotetizar, assim como encontrado na literatura, que o fato de praticar mais exercícios físicos é um fator protetivo para o estresse, pois há evidências que estudantes que praticam atividades físicas mais de uma vez por semana se mostram menos estressados (Fioravanti, Shayani, Borges, \& Balieiro, 2005).

Em relação à sintomatologia depressiva, os resultados expõem que a prevalência de sintomas depressivos esteve presente apenas na minoria dos participantes da pesquisa. Esse dado se aproxima com o encontrado em outros estudos (Brandtner \& Bardagi, 2009; Simić-Vukomanovićet al., 2016). Por outro lado, há estudo que indica um número elevado de sintomatologia depressiva em universitários, chamando atenção para um processo de reflexão e cuidado, uma vez que depressão pode evoluir para outros níveis, levando o sujeito ao suicídio (Paula et al., 2014).

De forma geral, o estudo apontou que a maioria dos estudantes apresenta algum sofrimento mental ou predisposição para tal. No entanto se faz necessário uma avaliação mais profunda para realização de um diagnóstico, tendo em vista que o SRQ-20 é um instrumento de rastreio, e não diagnóstico (Gonçalves et al., 2008; Santos, Araújo, Pinho, \& Silva, 2010). Apesar disso, os escores encontrados para sofrimento mental indicam que é necessária uma maior atenção para esses sujeitos, principalmente, por estarem em um ambiente de muita pressão. Lima, Domingues e Cerqueira (2006) encontraram resultados semelhantes aos desta pesquisa e atribuíram esse resultado ao fato de que cursos integrais, como é o caso dos analisados nesta pesquisa, exigirem maior empenho e dedicação, o que acarreta em redução no tempo para lazer. Conforme encontrado no presente estudo, a maioria dos estudantes afirma deixar de fazer coisas prazerosas por causa da universidade. A prevalência de sofrimento mental indica uma grave situação de saúde mental dos estudantes e aponta a necessidade da identificação precoce e intervenção adequada frente aos transtornos mentais comuns (Carleto, Moura, Santos, \& Pedrosa, 2018).

Durante a análise dos dados obtidos na pesquisa, pode-se observar a presença em maior ou menor grau de ansiedade, estresse, sintomatologia depressiva e sofrimento mental entre os estudantes. Apesar da presença desses índices, foi possível encontrar algumas informações que indicam presença de meios protetivos para esses estudantes. Como no caso dos dados encontrados a respeito dos estudantes que vivem em casa e não precisaram mudar de cidade para cursar a universidade. Almeida e Soares (2003) afirmam na sua pesquisa que a distância de casa poderia ser um agravador de sintomas ansiosos, depressivos e estressantes. Existem indícios sobre a importância do tipo de moradia do estudante universitário, pois, enquanto morar com a família pode beneficiar o bem-estar psicológico desse indivíduo, morar em moradias coletivas (como repúblicas e pensionato) podem representar fatores de risco à saúde mental (Rocha \& Sassi, 2013). Na presente pesquisa, foi encontrado que muitos moram com pessoas da família, incluindo pais, irmãos, companheiros, filhos ou outros parentes.

Outro fato importante para o enfrentamento desses fatores foi o elevado índice de contentamento desses com o curso. A esse respeito, Bardagi (2007) traz que índices elevados de satisfação com o curso colaboram para a saúde mental do sujeito. Ainda se pode destacar que, apesar do sofrimento verificado nesses estudantes, apenas a minoria buscou atendimento profissional. Cerchiariet, Caetano e Faccenda (2005) trazem em seu estudo que não buscar esse tipo de atendimento é uma condição comum à maioria dos universitários, podendo essa situação se prolongar ao longo do tempo, inclusive agravando os sintomas apresentados.

\section{Considerações Finais}

Os principais resultados encontrados neste estudo indicaram que não há diferença significativa entre a sintomatologia depressiva, ansiedade e estresse entre os estudantes ingressantes e concluintes. Quando comparado os índices por sexo, as mulheres apresentaram 
índices significativamente maiores que os homens, e os construtos estão altamente correlacionados. Foi encontrada uma alta prevalência de ansiedade entre os participantes (a maioria apresentou sintomatologia moderada e alta), comparando os índices de estresse por curso todos apresentaram níveis moderados; a minoria dos participantes apresentou sintomatologia depressiva e a maioria apresentou algum sofrimento mental ou predisposição para tal.

Diante dos resultados e discussões apresentadas até aqui se pode destacar a importância de discutir o tema de saúde mental no público universitário, que é também uma questão de saúde pública. Essa importância advém tanto da necessidade de se produzir novos conhecimentos, como também para servir de subsídio na elaboração de mecanismos de cuidado, como projetos que visem proporcionar melhoria na qualidade de vida desses universitários.

As universidades poderiam incentivar a promoção da saúde do universitário a partir de projetos vivenciados nos seus cursos, sendo realizados pelos próprios universitários (estudantes em estágio de Psicologia, Educação Física, Nutrição, por exemplo) assim como sendo esse público-alvo. Dessa forma, proporcionaria conhecimento e promoção de cuidado. Ainda poderiam ser realizados programas específicos para as mulheres, tendo em vista todas as suas peculiaridades e a maior prevalência nesse grupo.

Estudos futuros podem ser feitos para preencher as lacunas desta pesquisa. Esta pode apresentar limitações, no se que se refere à amostra utilizada. Um estudo mais detalhado poderia utilizar uma amostra que contemplasse outros períodos, assim como também estudos longitudinais que acompanhem o aluno da entrada até a saída da universidade. Também poderia se avaliar e comparar os estudantes de saúde com os de outras áreas. Além disso, é importante que novos estudos sejam realizados com os instrumentos utilizados, buscando evidências de validade, bem como realizando estudos que comparem os achados do presente estudo com instrumentos aprovados pelo SATEPSI (Sistema de Avaliação dos Testes Psicológicos) para uso profissional.

\section{Referências}

Agostini, P., Sakae, T. M., \& Feldens, V. P. (2010). Prevalência de sintomas ansiosos em pacientes em consultório de anestesia de Tubarão. Revista Arquivos Catarinenses de Medicina, 40(1), 52-56.
Recuperado de http://www.acm.org.br/revista/ pdf/artigos/847.pdf

Almeida, L. S., \& Soares, A. P. (2003). Os estudantes universitários: Sucesso escolar e desenvolvimento psicossocial. Em E. Mercuri \& S. A. J. Polydoro (Eds.), Estudante universitário: características e experiências de formação (pp. 15-40). Taubaté, SP: Cabral Editora.

Araújo, T. M, Pinho, P. S, \& Almeida, M. M. G. (2005). Prevalência de transtornos mentais comuns em mulheres e sua relação com as características sociodemográficas e o trabalho doméstico. Revista Brasileira de Saúde Materno Infantil, 5(3), 337-348. doi:10.1590/S1519-38292005000300010

Alves, T. C. T. F. (2014). Depressão e ansiedade entre estudantes da área de saúde. Revista de Medicina, 93(3), 101-105. doi:10.11606/issn.1679-9836. v93i3p101-105

Alvi, T., Assad, F., Ramzan, M., \& Khan, F. A. (2010). Depression, anxiety and their associated factors among medical students. Journal of the College of Physicians and Surgeons Pakistan, 20(2), 122-126. doi: 02.2010/JCPSP.122126

Apóstolo, J. L. A., Figueiredo, M. H., Mendes, A. C., \& Rodrigues, M. A. (2011). Depressão, ansiedade e estresse em usuários de cuidados primários de saúde. Revista Latino-Americana de Enfermagem, 19(2). Recuperado de http://www.scielo.br/pdf/rlae/ v19n2/pt_17.pdf

Assis, C. L., Silva, A. P. F., Lopes, M. S., Silva, P. C. B., \& Santini, T. O. (2013). Sintomas de estresse em concluintes do curso de psicologia de uma faculdade privada do norte do País. Mudanças - Psicologia da Saúde, 21(1), 23-28. doi:10.15603/2176-1019/mud. v21n1p23-28

Baptista, M. N., \& Gomes, J. O. (2011). Escala Baptista de Depressão (Versão Adulto) - EBADEP-A: Evidências de validade de construto e de critério. Psico-USF, 16(2), 151-161. Recuperado de http:/ / www.scielo.br/pdf/pusf/v16n2/v16n2a04.pdf

Bardagi, M. P. (2007). Evasão e comportamento vocacional de universitários: Estudos sobre o desenvolvimento de carreira na graduação (Tese de doutorado). Universidade Federal do Rio Grande do Sul, Porto Alegre-RS.

Brandtner, M., \& Bardagi, M. (2009). Sintomatologia de depressão e ansiedade em estudantes de uma 
universidade privada do Rio Grande do Sul. Revista Interinstitucional de Psicologia, 2(2), 81-91. Recuperado de http://pepsic.bvsalud.org/pdf/gerais/v2n2/v2n2a04.pdf

Biaggio, A. M. B., \& Natalício, L. (2003). Manual para o Inventário de Ansiedade Traço-Estado (IDATE). Centro Editor de Psicologia Aplicada-CEPA, Rio de Janeiro, RJ, Brasil.

Carleto, C. T., Moura, R. C. D., Santos, V. S., \& Pedrosa, L. A. K. (2018). Adaptação à universidade e transtornos mentais comuns em graduandos de enfermagem. Rev. Eletr. Enf. [Internet], 20(1). doi:10.5216/ree.v20.43888

Carvalho, E. A., Bertolini, S. M. M. G., Milani, R. G., \& Martins, M. C. (2015). Índice de ansiedade em universitários ingressantes e concluintes de uma instituição de ensino superior. Ciência, Cuidado e Saúde, 14(3), 1290-1298.doi:10.4025/cienccuidsaude.v14i3.23594

Cavestro, J. M., \& Rocha, F. L. (2006). Prevalência de depressão entre estudantes universitários. Jornal Brasileiro de Psiquiatria, 55(4), 264-267. doi:10.1590/ S0047-20852006000400001

Cerchiari, E. A. N., Caetano, D., \& Faccenda, O. (2005). Utilização do serviço de saúde mental em uma universidade pública. Psicologia: Ciência e Profissão, 25(2), 252-265. doi:10.1590/ S1414-98932005000200008

Draken, A. A. S., Jr, M. H., Rush, B. R., \& Reisbig, A. M. J. (2012). Predictors of anxiety and depression in veterinary medicine students: A four-year cohort examination. Journal of Veterinary Medical Education, 39(4), 322-330. doi: 10.3138/jvme.0112-006R

Figueiredo, A. M., Ribeiro, G. M., Reggiani, A. L. M., Pinheiro, B. A., Leopoldo, G. O., Duarte, J. A. H., Oliveira, L. B., \& Avelar, L. M. (2014). Percepções dos estudantes de medicina da UFOP sobre sua qualidade de vida. Revista Brasileira de Educação Médica, 38(4), 435-443. doi:10.1590/ S0100-55022014000400004

Fioravanti, A. R., Shayani, D. A., Borges, R. C., \& Balieiro, R. C. (2005). Estudo sobre os fatores de stress entre alunos da Unicamp. Revista Ciências do Ambiente[online], 1(1). Recuperado de http:// sistemas.ib.unicamp.br/be310/nova/index.php/ be310/article/view/21/9
Fioravanti, A. C. M., Santos, L. F., Maissonette, S., Cruz, A. P. M., \& Landeira-Fernandez, J. (2006). Avaliação da estrutura fatorial da escala de ansiedade-traço do IDATE. Avaliação Psicológica, 5(2), 217-224. Recuperado de http://pepsic.bvsalud. org/scielo.php?script $=$ sci_arttext\&pid $=\mathrm{S} 1677$ $-04712006000200011 \& \operatorname{lng}=\mathrm{pt \& t} \operatorname{lng}=$ $\mathrm{pt}$

Fonseca, A. A., Coutinho, M. P. L., \& Azevedo, R. L. W. (2008). Representações sociais da depressão em jovens universitários com e sem sintomas para desenvolver a depressão. Psicologia: Reflexão e Crítica, 21(3), 492-498. doi:10.1590/ S0102-79722008000300018

Gonçalves, D. M., Stein, A. T., \& Kapczinski, F. P. (2008). Avaliação de desempenho do Self-Reporting Questionnaire como instrumento de rastreamento psiquiátrico: Um estudo comparativo com o Structured Clinical Interview for DSM-IV-TR. Cadernos de saúde pública. Rio de Janeiro. 24(2), p. 380-390. doi:10.1590/S0102-311X2008000200017

Keita, P. G. (2007). Psychosocial and cultural contributions to depression in women: Considerations for women midlife and beyond. Journal of Managed Care Pharmacy, 13(9), 12-15. doi:10.18553/ jmcp.2007.13.9-a.12

Rodrigues, E. O. L., Marques, D. A., Neto, D. L., Montesinos, M. J. L., \& Oliveira, A. S. A. (2016). Situações e fatores de estresse em estudantes de enfermagem na prática clínica. Investigación y Educación en Enfermería, 34(1), 211-220. doi:10.17533/ udea.iee.v34n1a23

Lacerda, A. N. (2015). Indícios de estresse, ansiedade e depressão em estudantes universitários (Monografia). Universidade de Brasília, Brasília-DF. Recuperado de http://bdm.unb.br/handle/10483/12965

Lima, M. C. P., Domingues, M. S., \& Cerqueira, A. T. A. R. (2006). Prevalência e fatores de risco para transtornos mentais comuns entre estudantes de medicina. Revista de Saúde Pública, 40(6), 1035-1041. doi:10.1590/S0034-89102006000700011

Mancebo, D., Vale, A. A., \& Martins, T. B. (2015). Políticas de expansão da educação superior no Brasil 1995-2010. Revista Brasileira de Educaşão, 20(60), 31 50. doi:10.1590/S1413-24782015206003

Marchi, K. C., Bárbaro, A. M., Miasso, A. I., \& Tirapelli, C. R. (2013). Ansiedade e consumo de ansiolíticos 
entre estudantes de enfermagem de uma universidade pública. Revista Eletrônica de Enfermagem, 15(3), 729- 37. doi:10.5216/ree.v15i3.18924

Marques, A. C. H., Cepêda, V. A., \& Zambello, A. V. (2015, outubro). Mudanças no ensino superior no Brasil - Expansão, inclusão e equidade: um balanço do REUNI e a recepção de influências internacionais. In GT25:Novas configuraçōes do ensino superior na sociedade contemporânea. $39^{\circ}$ Encontro Anual da ANPOCS, Caxambú, MG, 1-17. Recuperado de https://anpocs.com/index.php/encontros/ papers/39-encontro-anual-da-anpocs/gt/gt25

Martincowski, T. M. (2013). A inserção do aluno iniciante de graduação no universo autoral: A leitura interpretativa e a formação de arquivos. Cadernos da Pedagogia. São Carlos, 6(12), p. 129-140. Recuperado de http://www.cadernosdapedagogia.ufscar. br/index.php/cp/article/view/508/209

Molina, M. A. L., Jansen, K., Drews, C., Pinheiro, R., Silva, R., \& Souza, L. (2014). Major depressive disorder symptoms in male and female young adults. Psychology, Health \& Medicine, 19(2), 136-145. do i:10.1080/13548506.2013.793369

Monteiro, S. O. M, Tavares, J. P. C., \& Pereira, A. M. S. (2008). Optimismo disposicional, sintomatologia psicopatológica, bem-estar e rendimento académico em estudantes do primeiro ano do ensino superior. Estudos de Psicologia (Natal), 13(1), 23-29. doi:10.1590/S1413-294X2008000100003

Neves, C. E. B., Raizer, L., \& Fachinetto, R. F. (2007). Acesso, expansão e equidade na educação superior: Novos desafios para a política educacional brasileira. Sociologias, (17), 124-157.doi:10.1590/ S1517-45222007000100006

Oliveira, E. N. (2013). Prevalência de sintomas depressivos em estudantes de Medicina da Universidade Federal da Babia (Monografia). Universidade Federal da Bahia, Salvador-BA. Recuperado de https://repositorio.ufba.br/ri/bitstream/ri/13959/1/ Elis\%C3\%A2ngela\%20Neves\%20de\%20Oliveira. pdf

Organização Mundial da Saúde. (2017). Aumenta o número de pessoas com depressão no mundo. Recuperado de http://www.paho.org/bra/index. php?option $=$ com_content\&view $=$ article $\&$ id $=535$ 4:aumenta-o-numero-de-pessoas-com-depressao-no-mundo\&Itemid $=839$
Padovani, R. C., Neufeld, C. B., Maltoni, J., Barbosa, L. N. F., Souza, W. F., Cavalcanti, H. A. F., \& Lameu, J. N. (2014). Vulnerabilidade e bem-estar psicológicos do estudante universitário. Revista Brasileira de Terapias Cognitivas. 10(1), 2-10. doi:10.5935/1808-5687.20140002

Paula, J. A., Borges, A. M, F. S., Bezerra, L. R. A., Parente, H. V., Paula, R. C. A., Wajnsztejn, R., Carvalho, A. A. S., Valenti, V. E., \& Abreu, L. C. (2014). Prevalência e fatores associados à depressão em estudantes de medicina. Journal of Human Growth and Development, 24(3), 274-281. Recuperado de http:// pepsic.bvsalud.org/scielo.php?script $=$ sci_ arttext\&pid=S0104-12822014000300006\&lng=pt \&tlng $=\mathrm{pt}$

Pinho, P. S., \& Araújo, T. M. (2012). Associação entre sobrecarga doméstica e transtornos mentais comuns em mulheres. Revista Brasileira de Epidemiologia, 15(3), 560-572.doi:10.1590/S1415-790X2012000300010

Ramos, A. M., Barlem, J. G. T., Lunardi, V. L., Barlem, E. L. D., Silveira, R. S., \& Bordignon, S. S. (2015). Satisfaction with academic experience among undergraduate nursing students. Texto \& Contexto - Enfermagem, 24(1), 187-195. doi:10.1590/0104-07072015002870013

Reis, R. S., Hino, A. A. F., \& Añez, C. R. R. (2010). Perceived stress scale: Reliability and validity study in Brazil. Journal of health psychology, 15(1), 107-114. doi:10.1177/1359105309346343

Rocha, E. S., \& Sassi, A. P. (2013). Transtornos mentais menores entre estudantes de medicina. Revista BrasiLeira de Educação Médica, 37(2), 210-216. Recuperado de http://www.scielo.br/scielo.php?script=sci_ arttext\&pid=S0100-55022013000200008\&lng=en $\& \operatorname{tlng}=\mathrm{pt}$

Santos, T. M., Almeida, A. O., Martins, H. O., \& Moreno, V. (2003). Aplicação de um instrumento de avaliação do grau de depressão em universitários do interior paulista durante a graduação de enfermagem. Acta Scientarium. Health Sciences, 25(2), 171-176. doi:10.4025/actascihealthsci. v25i2.2228

Santos, K. O. B., Araújo, T. M., Pinho, P. S., \& Silva, A. C. C. (2010). Avaliação de um instrumento de mensuração de morbidade psíquica: Estudo de validação do Self-Reporting Questionnaire (SRQ-20). Revista Baiana Saúde Pública Miolo,34(3), 
544-560. Recuperado de http://files.bvs.br/ upload/S/0100-0233/2010/v34n3/a1881.pdf

Santos, K. A. S., Cendoroglo, M. S., \& Santos, F. C. (2017). Transtorno de ansiedade em idosos com dor crônica: Frequência e associações. Revista Brasileira de Geriatria e Gerontologia, 20(1), 91-98. Recuperado de http://www.scielo.br/scielo.php?script=sci_ arttext\&pid=S1809-98232017000100091\&lng=en $\& n r m=$ iso\&tlng $=$ pt

Simić-Vukomanović, I., Mihajlović, G., Kocić, S., Djonović, N., Banković, D., Vukomanović, V., \& Djukić-Dejanović, S. (2016). The prevalence and socioeconomic correlates of depressive and anxiety symptoms in a group of 1,940 Serbian university students. Vojnosanitetski Pregled, 73(2), 169-177. doi:10.2298/VSP141106143S

Sousa, T. F., José, H. P. M., \& Barbosa, A. R. (2013). Condutas negativas à saúde em estudantes universitários brasileiros. Ciência \& Saúde Coletiva, 18(12), 35633575. doi:10.1590/S1413-81232013001200013

Vasconcelos, T. C., Dias, B. R. T., Andrade, L. R., Melo, G. F., Barbosa, L., \& Souza, E. (2015). Prevalência de Sintomas de Ansiedade e Depressão em Estudantes de Medicina. Revista Brasileira de Educação Médica, 39(1), 135-142. doi:10.1590/1981-52712015v39n1e00042014

Vieira, L. N., \& Schermann, L. B. (2015). Estresse e fatores associados em alunos de psicologia de uma universidade particular do sul do Brasil. Aletheia, (46), 120-130. Recuperado de http:// pepsic.bvsalud.org/scielo.php?script $=$ sci arttext\&pid=S1413-03942015000100010\&lng=pt \&tlng $=\mathrm{pt}$

Recebido em: 12/11/2018

Reformulado em: 24/07/2019

Aprovado em: 19/11/2019

Sobre as autoras:

Marília Guimarães Leal Jardim é Psicóloga e Bacharela em Administração pela Universidade Federal do Vale do São Francisco

ORCID: https://orcid.org/0000-0001-7914-8339

E-mail:mariliagljardim@gmail.com

Tathyane Silva Castro é Psicóloga pela Universidade Federal do Vale do São Francisco.

ORCID: https://orcid.org/0000-0003-1667-2744

E-mail: tathiane.castro@hotmail.com

Carla Fernanda Ferreira-Rodrigues é Doutora em Psicologia com área de concentração em Avaliação Psicológica pela Universidade São Francisco. Docente do Colegiado de Psicologia e do Programa de Pós-Graduação Stricto Sensu da Universidade Federal do Vale do São Francisco.

ORCID: http://orcid.org/0000-0002-6590-1681

E-mail: carla.fernanda@univasf.edu.br

Contato com as autoras:

Universidade Federal do Vale do São Francisco

Colegiado de Psicologia

Av. José de Sá Maniçoba S/N, Centro

Petrolina-PE, Brasil

CEP: 56304-917

Psico-USF, Bragança Paulista, v. 25, n. 4, p. 645-657, out./ dez. 2020 